\title{
Effects of rhBNP on Elderly Emergency Patients with Acute Heart Failure
}

\author{
Wang J, Li Y*, Guo J and Nie S \\ Department of Emergency, Central Hospital of Cangzhou City, Cangzhou 061001, Hebei, China
}

*Corresponding author: Li Y, Department of Emergency, Central Hospital of Cangzhou City, Cangzhou 061001, Hebei, China, Tel: 0317-2022433; Email: xiaozhuhetang@126.com

Received date: September 22, 2016; Accepted date: January 19, 2018; Published date: January 25, 2018

Citation: Wang J, Li Y, Guo J, Nie S (2018) Effects of rhBNP on Elderly Emergency Patients with Acute Heart Failure. Arch Med Vol No:10 Iss No:1:3

Copyright: (c) 2018 Wang J, et al. This is an open-access article distributed under the terms of the Creative Commons Attribution License, which permits unrestricted use, distribution, and reproduction in any medium, provided the original author and source are credited.

\section{Abstract}

Objective: To study the effect of re-combinant human brain natriuretic peptide (rhBNP) in older emergency patients with acute heart failure.

Patients and Methods: A total of 130 patients were randomly divided into two groups as: rhBNP treatment group and sodium nitroprusside (SNP) treatment group, half by half. The rhBNP was taken once every three days for treatment. But dose was doubled at first time used. In the whole progress, it measured the concentration of rhBNP. Echocardiography examination and 6-minute walk testing (6MWT) were used to evaluate the improvement of heart function after rhBNP treatment, compared with SNP.

Results: It taken vital symptoms assessment according to the clinical manifestations, once every four hours in the whole 48 hours emergency treatment procedure. Compared before and after treatment, there were significant improvement about LVEF (left ventricular ejection fraction), 6MWT and proBNP level changes in rhBNP group. Furthermore, it revealed that there were also significant differences in LVEF, 6MWT and proBNP level changes between rhBNP and SNP group.

Conclusion: Recombinant human brain natriuretic peptide (rhBNP) could be used to treat old patients with acute heart failure in emergency with meaningful clinical value.

Keywords: rhBNP; Treatment; Acute heart failure; Emergency

\section{Introduction}

Brain natriuretic peptide is a 32 -amino acid poly peptide secreted by the ventricles of the heart in response to excessive stretching of heart muscle cells (cardiomyocytes) [1,2]. Recombinant human brain natriuretic peptide (rhBNP) possesses the same amino acid sequence and mode of action with endogenous peptides-brain natriuretic peptide (BNP) secreted by ventricular myocytes $[3,4]$.
The main clinical function of BNP is to keep a normal level for acute heart failure in the emergency setting [5,6]. However, an elevated BNP should never be used to "rule in" acute or chronic heart failure in the emergency setting due to lack of specificity, dubious-discuss [7].

In recent study, BNP increases PV arrhythmogenesis, which may contribute to the genesis of atrial tachyarrhythmogenesis. Cyclic GMP activation, phosphodiesterase 3 inhibition and $\mathrm{Na}+/ \mathrm{K}$ +-ATPase inhibition might participate in the BNP modulation of PV electrophysiology.

On the other hand, BNP can be used for screening and prognoses of heart failure. Both of them are also typically increased in patients with left ventricular dysfunction, with or without symptoms [8].

Recombinant human B-type natriuretic peptide (rhBNP) could bind the natriuretic peptide $A$ receptor (NPR-A), which decreased the pulmonary capillary wedge pressure (PCWP) [9]. That innate renin-angiotensin-aldosterone system (RAAS) increased cardiac output and urinary output and improved heart diastolic function [10]. rhBNP is used to treat decompensate heart failure. However, a recent clinical trial failed to show a benefit of nesiritide in patients with acute decompensate heart failure, and the authors could not recommend its use. There was no publication and less study about rhBNP affected acute heart failure in Emergency Department.

\section{Materials and Methods}

\section{Study population}

A total of 130 subjects were from the Emergency Department of Cangzhou Center Hospital, Hebei Medical University, from June 2013 to December 2015. The population were 67-male and 63 -female, and their ages were from 43 to 66 year-old (average age at 57). All subjects were divided into rhBNP group (treated with rhBNP) and SNP group (treated with Sodium nitroprusside) randomly by ratio of $1: 1$

\section{Case definition}

After ECG examination, they could be diagnosed as acute heart failure, if patients suffered one of these four symptoms 
(with new chemist electrocardiography changes; new ST-T changes; new left bundle branch block; or prompted the formation of pathological $Q$ wave). And they suffered from myocardial infarction associated with heart failure (Killip class to ).

Blood pressure was measured, through controlling systolic blood pressure (SBP) above $90 \mathrm{mmHg}$ and diastolic blood pressure (DBP) above $60 \mathrm{mmHg}$. All subjects didn't have complication of coronary heart disease, high blood pressure, dilated cardiopulmonary and pulmonary heart disease. The exclusion criteria for respondents were including cardinal shock, hypernatremia, valvular stenosis, restrictive cardiomyopathy, pericarditis and abnormal liver function.

All study participants provided written informed consent. This study was according to the declaration of Helsinki and world health organization guidelines to implement. It was under the supervision of the Ethics Committee of the Hebei Province Medical University.

\section{Therapy methods}

Control group was treated with the standard treatment of heart failure, including limit salt, diuretics and angiotensinconverting enzyme inhibitors (ACEI), which was purchased from Sigma Corporation of America. Intravenous dose were $4 \mathrm{mg}$ per $\mathrm{kg}$, flowing rate from $20 \mu \mathrm{g} / \mathrm{min}$ to $100 \mu \mathrm{g} / \mathrm{min}$.

As for rhBNP group, treated the patients with lyophilized recombinant human brain natriuretic peptide (rhBNP), which were purchased from China medical system holdings Ltd. Intravenous dose were $1.5 \mu \mathrm{g} / \mathrm{kg}$, following the flow rate of 0.0075 to $0.020 \mu \mathrm{g} / \mathrm{kg} / \mathrm{min}$. But dose was doubled at first time used. Besides, it ensured the systolic blood pressure above 90 $\mathrm{mmHg}$, during treatment. This treatment process should be carried at least in 2 hours, which taken once every three days.

Simultaneously, medical observation for all patients was implemented for difficulty breathing, and other clinical symptoms. Tested rhBNP concentration and taken electrocardiograph examination after 3 days treatment. 6minute walk testing (6MWT) were used to exam the live capacity, for each patient.
Because of severity of disease, all patients were permitted that received other drug treatment, including aspirin, clopidogrel, stains, diuretics, morphine and other vasoactive medications, according to standard heart failure therapies. These medical treatments didn't constitute confounding factors for this study.

\section{Ultrasonic inspection}

It was following that progress: electrocardiograph was taken within 4 hours in emergency department. Tested whether ultrasonic wave was increased to $25 \%$, it decided how to taken emergency measures. All patients were tested the left ventricular ejection fraction (LVEF), Left ventricular diameter (LVDD).

\section{Statistical analysis of data}

All data are represented as means \pm SD $(\bar{x} \pm s)$ of three or more independent experiments. The data are changed into normal distribution with logarithm if the original data are positive skewness distribution. Comparisons among the experimental groups, and the correlations between two groups were analyzed by chi-square test or Fisher exact test. All the analyses were carried out using the SPSS19.0 software (SPSS Inc., Chicago, IL, USA). Values less than 0.05 were considered to be statistically significant.

\section{Results}

\section{Population}

This research population was composed of 130 cases of acute heart failure. Table 1 summarized the epidemiological characteristics of rhBNP and SNP group. Of 130 patients, 67 were male and 63 were female, with an average male-to-female sex ratio of 1.06. The average age was 57 years, from 46 to 73 years. The average of left ventricular ejection fraction (LVEF) was $48.17 \%$. And the baseline distributions of Killip classification were $83.51 \%$ at stage and $16.49 \%$ at stage . All patients were in the classical clinical system of acute heart failure. The selection for this study was eligible.

Table 1: Basic demographics and clinical characteristics.

\begin{tabular}{|c|c|c|c|}
\hline Characteristic & rhBNP group $(n=65)$ & SNP group $(n=65)$ & $p$ value \\
\hline Age (years) & $51.48 \pm 9.81$ & $52.64 \pm 8.83$ & 0.779 \\
\hline Sex (male) & $39(60.0 \%)$ & $40(61.5 \%)$ & 0.813 \\
\hline BMI $\left(\mathrm{kg} / \mathrm{m}^{2}\right)$ & $27.16 \pm 4.42$ & $26.78 \pm 3.77$ & 0.62 \\
\hline Diabetes $(\mathrm{n} /(\%))$ & $18(31.58 \%)$ & $15(25.42 \%)$ & 0.597 \\
\hline Hypertension (n/(\%)) & $31(54.39 \%)$ & $35(59.32 \%)$ & 0.728 \\
\hline LVEF (\%) & $44.95 \pm 7.80$ & $47.43 \pm 7.20$ & 0.077 \\
\hline
\end{tabular}

BMI: Body Mass Index; LVEF: Left Ventricular Ejection Fraction; BUN: Blood Urea Nitrogen; EGFR: Estimate Glomerular Filtration Rate; ACEI: Angiotensin-Converting Enzyme Inhibitors; ARB: Angiotensin Receptor Blockers; CCB: Calcium Channel Blockers. 


\section{Determination of the clinical efficacy of rhBNP}

The difficulty breathing was classified as four grades: supine, paroxysmal nocturnal, semi-recumbent and orthopnea. Before and after treatment, emergency physicians made the score according to the clinical symptoms. Table 2 summarized the clinical efficacy of rhBNP before and after therapy 3 days in each group. It could be found that rhBNP might effectively improve the heart function, immediately.

Table 2: Evaluation of the clinical efficacy of the two groups after therapy.

\begin{tabular}{|l|c|c|c|c|c|}
\hline Groups & $\mathbf{n}$ & significant effective & Normal effective & Ineffective & $70.8 \%{ }^{*}$ \\
\hline SNP group & 65 & 20 & 26 & $49.8 \%$ \\
\hline rhBNP group & 65 & 36 & 25 & 4 \\
\hline \multicolumn{2}{|l}{ Note: *With significant difference, $p<0.05}$.
\end{tabular}

\section{Monitoring of heart function}

After echocardiography and 6MWT testing, compared the difference of effectiveness improvement between rhBNP and SNP group. Compare before and after treatment, there were significant differences for LVEF, 6MWT and proBNP level changes in the each group. It illustrated that rhBNP and sodium nitroprusside could be used to treat with acute heart failure in Table 3. Further analysis, it revealed that there were also significant differences for LVEF, 6MWT and proBNP level changes between rhBNP and SNP group. Importantly, the advantage of rhBNP was more obvious.

Table 3: Comparison of heart function between rhBNP group and SNP group.

\begin{tabular}{|c|c|c|c|c|c|}
\hline \multicolumn{2}{|l|}{ Group } & $\operatorname{LVDD}(\mathrm{mm})$ & LVEF (\%) & 6MWT (m) & proBNP (pg/ml) \\
\hline \multirow{2}{*}{ SNP group } & Before treatment & $65.8 \pm 3.7$ & $35.4 \pm 6.5$ & $299.1 \pm 66.5$ & $7646.1 \pm 917.2$ \\
\hline & After treatment & $64.3 \pm 4.1$ & $48.8 \pm 5.4^{*}$ & $387.3 \pm 50.7^{*}$ & $7063.3 \pm 941.1^{*}$ \\
\hline \multirow{2}{*}{ rhBNP group } & Before treatment & $67.1 \pm 3.3$ & $36.2 \pm 5.5$ & $287 \pm 60.9$ & $7658.6 \pm 911.5$ \\
\hline & After treatment & $63.5 \pm 4.4$ & $51.1 \pm 4.7^{*}, \#$ & $403 \pm 59.7^{*}, \#$ & $6039.5 \pm 818.6^{*}$ \\
\hline
\end{tabular}

\section{Safety evaluation}

According to the results of three-month follow-up, there was no significant difference between the two groups, and observed all-cause mortality, which compared with nitroglycerin group in rate of increase or decrease about readmission and mortality within 3 months follow-up.

\section{Discussion}

rhBNP relaxes vascular smooth muscle in arterioles and venues by: membrane receptor-mediated elevation of vascular smooth muscle cGMP [10].

rhBNP also as known as neprilysin is the enzyme that metabolizes natriuretic peptides. Several inhibitors of NEP are currently being developed to treat disorders ranging from hypertension to heart failure. Most of them are dual inhibitors. In 2014, meaningful study was published in NEJM. This study considered as a landmark study in treatment of heart failure. The study was compared rhBNP with enalapril in patients with heart failure, by double blinded, which showed lower all cause mortality, cardiovascular mortality and hospitalization in rhBNP group [11].

This study was to evaluate the effect of rhBNP in Emergency Department for older heart failure patients. After survey, we suggested that rhBNP could be priority using, because heart failure was characterized by insufficient cardiac output, with consequences inadequate perfusion for peripheral demands $[12,13]$.

Since BNP was implication of ventricular wall if heart's ventricles were dilated, deterioration of clinical features increased or exercise capability decreased [11]. Patients with acute heart failure usually require more addition of rhBNP to relief of congestion. The recombinant of brain natriuretic peptide (rhBNP) was approved in 2001 by the US Food and Drug Administration (FDA) for the treatment of decompensates heart failure. The rhBNP could reduce cardiac stress, which were through vasodilatory properties, diuresis,and improvement inotropic arrhythmias [14].

Previous studies have demonstrated that rhBNP might result in better cardiorenal hemodynamics, which increased cardiac output and renal function and restrained neurohormonal mediators function. So in our study, rhBNP could be used for decompensate heart failure patients.

\section{Conflict of Interest}

We confirm that the manuscript has been read and approved by all named authors and that there are no other persons who 
satisfied the criteria for authorship but are not listed. We further confirm that the order of authors listed in the manuscript has been approved by all of us. We confirm that we have given due consideration to the protection of intellectual property associated with this work and that there are no impediments to publication, including the timing of publication, with respect to intellectual property. In so doing we confirm that we have followed the regulations of our hospital concerning intellectual property.

\section{References}

1. Song Z, Zhao X, Liu M, Jin H, Cui Y, et al. (2016) Recombinant human brain natriuretic peptide attenuates LPS-induced cellular injury in human fetal lung fibroblasts via inhibiting MAPK and NFKB pathway activation. Mol Med Rep 14: 1785-1790.

2. Su Z, Wei G, Wei L, Liu J, Li X (2015) Effects of rhBNP on myocardial fibrosis after myocardial infarction in rats. Int J Clin Exp Pathol 8: 6407-6415.

3. Zhou FJ, Zhou CY, Tian YJ, Xiao AJ, Li PL, et al. (2014) Diagnostic value of analysis of $\mathrm{H}-\mathrm{FABP}, \mathrm{NT}-\mathrm{proBNP}$, and $\mathrm{cTnl}$ in heart function in children with congenital heart disease and pneumonia. Eur Rev Med Pharmacol Sci 18: 1513-1516.

4. Chen K, Jiang RJ, Wang CQ, Yin ZF, Fan YQ, et al. (2013) Predictive value of plasma galectin-3 in patients with chronic heart failure. Eur Rev Med Pharmacol Sci 17: 1005-1011.

5. Zhang DL, Xu QZ (2013) Study of the recombinant human brain natriuretic peptide in patients with acute heart failure patients. Chin J Mod Drug 7: 97-98.

6. He XM, Chen L, Luo JB, Feng XX, Zhang YB, et al. (2016) Effects of rhBNP after $\mathrm{PCl}$ on non-invasive hemodynamic in acute myocardial infarction patients with left heart failure. Asian Pac J Trop Med 9: 791-795.

7. Xiao J, Deng SB, She Q, Li J, Kao GY, et al. (2016) Allopurinol ameliorates cardiac function in non-hyperuricaemic patients with chronic heart failure. Eur Rev Med Pharmacol Sci 20: 756-761.

8. Lv MY, Deng SL, Long XF (2016) Retraction. rhBNP therapy can improve clinical outcomes and reduce in-hospital mortality compared with dobutamine in heart failure patients: A metaanalysis. Br J Clin Pharmacol 81: 174-185.

9. Wang Y, Gu X, Fan W, Fan Y, Li W, et al. (2016) Effects of recombinant human brain natriuretic peptide on renal function in patients with acute heart failure following myocardial infarction. Am J Transl Res 8: 239-245.

10. O'Connor CM, Starling RC, Hernandez AF, Armstrong PW, Dickstein $K$, et al. (2011) Effect of nesiritide in patients with acute decompensated heart failure. N Engl J Med 365: 32-43.

11. McMurray JJ, Packer M, Desai AS, Gong J, Lefkowitz MP, et al (2014) Angiotensin-neprilysin inhibition versus enalapril in heart failure. N Engl J Med 371: 993-1004.

12. Yang $H$, Song $Z$, Jin $H$, Cui $Y$, Hou M, et al. (2014) Protective effect of rhBNP on intestinal injury in the canine models of sepsis. Int Immunopharmacol 19: 262-266.

13. Zhu XQ, Hong HS, Lin XH, Chen LL, Li YH (2014) Changes in cardiac aldosterone and its synthase in rats with chronic heart failure: An intervention study of long-term treatment with recombinant human brain natriuretic peptide. Braz J Med Biol Res 47: 646-654.

14. Kose M, Akpinar TS, Bakkaloglu OK, Tufan A, Sumnu A, et al. (2014) Association of genetic polymorphisms with endothelial dysfunction in chronic heart failure. Eur Rev Med Pharmacol Sci 18: 1755-1761. 